\title{
РАЗДЕЛЕНИЕ ПАРАМЕТРА РАСТВОРИМОСТИ САМОАССОЦИИРОВАННЫХ СОЕДИНЕНИИ НА СОСТАВЛЯЮЩИЕ
}

\author{
(Представил О. Эйзен)
}

Для описания свойств растворов известно много различных подходов, в большей или меньшей степени основанных на фундаментальных представлениях о взаимодействии молекул в жидком состоянии. Из них одним из наиболее простых можно считать теорию регулярных растворов Скэтчарда-Гильдербранда (см., напр., [']), которая в своей классической форме базируется на концепции симметрической функции межмолекулярного потенциала. Эта модель успешно применяется для растворов неполярных соединений, причем обычно выражение для свободной энергии получают, принимая энтропию смешения идеальной или учитывая комбинаторную энтропию, обусловленную различием в размерах молекул, согласно теориям Флори-Хаггинса, Гуггенгейма-Ставермана и др. $\left[{ }^{1,2}\right]$.

В более сложных случаях, когда между молекулами действуют не только дисперсионные, но и ориентационные и индукционные силы, а также водородная связь, правило геометрического среднего для определения параметра взаимодействия между молекулами различных соединений явно неприменимо.

Тем не менее простота основного уравнения теории регулярных растворов наводила многих авторов на идею расширить эту теорию и на системы, которые в классическом понятии никак нельзя считать регулярными. Поэтому разработано несколько модификаций первоначальной теории, особенно для разбавленных растворов. В некоторых модификациях (напр., $\left[{ }^{3,4}\right]$ ) вводится один или больше полуэмпирических параметров. В большинстве случаев $\left[{ }^{5-12}\right]$ модификация теории заключается в разделении общей когезионной энергии чистой жидкости на составляющие, учитывающие различные виды межмолекулярного взаимодействия. ${ }^{*}$ Обычно используют трех- $\left[{ }^{8,9}\right]$ или четырехпараметрические ${ }^{\left.1{ }^{10-12}\right]}$ модели, в которых, как правило, одна составляющая характеризует вклад водородной связи.

В модификации, предложенной в $\left[{ }^{10}\right]$ и использованной в $\left[{ }^{13,14}\right]$ для описания систем без водородной связи, общая когезионная энергия единицы объема чистой полярной жидкости $\left(E^{c}\right)$ рассматривается как сумма четырех составляющих, характеризующих дисперсионные $\left(E_{d}^{c}\right)$,

* Иногда этот подход формулируется как рассмотрение параметра взаимообмена компонентов раствора в виде суммы нескольких составляющих. Легко убедиться, что обе трактовки приводят к абсолютно одннаковым результатам. 
ориентационные $\left(E_{o r}^{c}\right)$, индукционные $\left(E_{i n}^{c}\right)$ силы и водородную связь $\left(E_{h}^{\mathrm{c}}\right)$ :

$$
E^{c}=E_{d}^{c}+E_{o r}^{c}+E_{i n}^{c}+\dot{E}_{h}^{c} .
$$

При этом плотность когезионной энергии определяется через т. н. параметр растворимости $\delta$ :

$$
-E^{c} \equiv \delta^{2}=\left(\Delta H^{v}-R T\right) / V,
$$

где $\Delta H^{V}-$ мольная энтальпия испарения при температуре $T, V-$ мольный объем.

В соответствии с определением общей плотности когезионной энергии через суммарный параметр растворимости составляющие $E^{c}$ можно выразить через компоненты параметра растворимости так, чтобы выполнялись соотношения:

$$
-E_{d}^{c}=\delta_{d}^{2}, \quad-E_{o r}^{c}=\delta_{o r}^{2}, \quad-E_{i n}^{c}=2 \delta_{i n} \delta_{d}, \quad-E_{h}^{c}=2 \delta_{a} \delta_{b},
$$

где $\delta_{d}, \delta_{o r}, \delta_{i n}, \delta_{a}$ и $\delta_{b}-$ соответственно дисперсионная, ориентационная, индукционная, протонодонорная («кислотная») и протоноакцепторная («основная») составляющие $\delta$.

Тогда для чистой жидкости, ассоциированной посредством водородной связи, имеем

$$
\delta^{2}=\delta_{d}^{2}+\delta_{o r}^{2}+2 \delta_{i n} \delta_{d}+2 \delta_{a} \delta_{b} .
$$

Такая модель позволяет в принципе предсказать термодинамическое поведение растворов ассоциированных соединений, если для чистых компонентов раствора известны составляющие параметры растворимости, а также имеется информация (экспериментальная или чисто теоретическая). об избыточных энтропиях смешения.

Тем не менее, несмотря на привлекательность такого подхода, его практическое использование нередко затруднено вследствие отсутствия надежных методов определения составляющих $\delta$. Особенно это относится к $\delta_{a}$ и $\delta_{b}$.

В настоящей работе на примере растворов алифатических спиртов предлагается метод разделения суммарного параметра растворимости на составляющие исходя из экспериментальных данных о предельных коэффициентах активности в неполярных растворителях и парциальных энтальпиях растворения в воде.

\section{Общая модель}

Рассмотрим раствор вещества $B$ в растворителе $S$. Тогда, согласно ['], при аддитивности объемов при смешении $\left(V^{E}=0\right)$ избыточная свободная энергия Гиббса $\left(G^{E}\right)$ одного моля раствора выражается как

$$
G^{E}=\left(x_{B} V_{B}+x_{S} V_{S}\right) \varphi_{B} \varphi_{S} X_{B S}-T S^{E},
$$

где $x$ - мольная доля, $\varphi$ - объемная доля, $X$ - параметр взаимообмена, $S^{E}$ - избыточная энтропия, индексы $B$ и $S$ относятся к соответствующим компонентам раствора.

Представив параметр взаимообмена $X_{B S}$ в виде суммы компонентов, отражающих различные виды межмолекулярного взаимодействия

$$
X_{B S}=X_{d B S}+X_{o r B S}+X_{i n B S}+X_{h B S},
$$

получим вклады отдельных видов взаимодействия в $G^{E}$. 
Избыточную энергию, обусловленную дисперсионными силами $\left(E_{d}^{E}\right)$, можно записать в виде

$$
E_{d}^{E}=\left(x_{B} V_{B}+x_{S} V_{S}\right) \varphi_{B} \varphi_{S}\left(\delta_{d B}-\delta_{d S}\right)^{2} .
$$

Аналогично, избыточная энергия, обусловленная ориентацией взаимодействующих молекул $\left(E_{o r}^{E}\right)$, равна

$$
E_{o r}^{E}=\left(x_{B} V_{B}+x_{S} V_{S}\right) \varphi_{B} \varphi_{S}\left(\delta_{o r B}-\delta_{o r S}\right)^{2} .
$$

Избыточная энергия, обусловленная несимметричным взаимодействием диполей, индуцированных средой $\left(E_{i n}^{E}\right)$, может быть выражена как

$$
E_{i n}^{E}=2\left(x_{B} V_{B}+x_{S} V_{S}\right) \varphi_{B} \varphi_{S}\left(\delta_{i n B}-\delta_{i n s}\right)\left(\delta_{d B}-\delta_{d S}\right),
$$

где множитель $\left(\delta_{d B}-\delta_{d S}\right)$ характеризует разность в поляризуемости компонентов $B$ и $S$.

Если компоненты $B$ и $S$ взаимодействуют путем образования водородной связи, то избыточная энергия, обусловленная этим взаимодействием $\left(E_{h}^{E}\right)$, равна

$$
E_{h}^{E}=2\left(x_{B} V_{B}+x_{S} V_{S}\right) \varphi_{B} \varphi_{S}\left(\delta_{a B}-\delta_{a S}\right)\left(\delta_{b B}-\delta_{b S}\right) .
$$

Нетрудно убедиться, что при таком определении $E_{i n}^{E}$ и $E_{h}^{E}$ могут быть как положительными, так и отрицательными, а $E_{d}^{E}$ и $E_{o r}^{E}-$ только положительными величинами.

Общая избыточная энергия Гиббса одного моля раствора выражается как

$$
\begin{gathered}
G^{E}=\left(x_{B} V_{B}+x_{S} V_{S}\right) \varphi_{B} \varphi_{S}\left[\left(\delta_{d B}-\delta_{d S}\right)^{2}+\left(\delta_{o r B}-\delta_{o r S}\right)^{2}+\right. \\
\left.+2\left(\delta_{i n B}-\delta_{i n s}\right)\left(\delta_{d B}-\delta_{d S}\right)+2\left(\delta_{a B}-\delta_{a S}\right)\left(\delta_{b B}-\delta_{b S}\right)\right]-T S^{E} .
\end{gathered}
$$

Если растворитель $S$ является неполярным веществом, то $\delta_{o r} s=0$, $\delta_{i n s}=0, \delta_{a} s=0, \delta_{b s}=0$ и $\delta_{d s} \equiv \delta s$. Тогда уравнение (10) упрощается:

$$
G^{E}=\left(x_{B} V_{B}+x_{S} V_{S}\right) \varphi_{B} \varphi_{S}\left(\delta_{B}^{2}+\delta_{S}^{2}-2 \delta_{d B} \delta_{S}-2 \delta_{i n B} \delta_{S}\right)-T S^{E} .
$$

Парциальные избыточные свободные энергии Гиббса для компонентов $B$ и $S$ (соответственно $\bar{G}_{B}^{E}$ и $\bar{G}_{\mathrm{s}}^{E}$ ) получаем путем дифференцирования функции $G^{E}$ по числу молей компонентов $n_{B}$ и $n_{S}$ соответственно. Принимая, что избыточная энтропия смешения обусловлена только различием в размерах молекул и выражается уравнением Флори-Хаггинса [']

$$
S^{E}=-R\left(x_{B} \ln \frac{\varphi_{B}}{x_{B}}+x_{S} \ln \frac{\varphi_{S}}{x_{S}}\right),
$$

для бесконечно разбавленного раствора $B$ в $S\left(x_{B} \rightarrow 0\right)$ можно записать

$$
\begin{gathered}
\bar{G}_{B}^{E} \equiv R T \ln \gamma_{B(S)}^{\infty}=V_{B}\left[\left(\delta_{d B}-\delta_{d S}\right)^{2}+\left(\delta_{o r B}-\delta_{o r s}\right)^{2}+\right. \\
\left.+2\left(\delta_{i n B}-\delta_{i n s}\right)\left(\delta_{d B}-\delta_{d S}\right)+2\left(\delta_{a B}-\delta_{a S}\right)\left(\delta_{b B}-\delta_{b S}\right)\right]+ \\
+R T\left(\ln \frac{V_{B}}{V_{S}}+1-\frac{V_{B}}{V_{S}}\right)
\end{gathered}
$$

где $\gamma_{B(S)}^{\infty}-$ коэффициент активности $B$ в $S$ при бесконечном разбавлении. 
Аналогично, из уравнения (11) для раствора $B$ в непіолярном $S$ получим

$$
\begin{aligned}
R T \ln \gamma_{B(S)}^{\infty} & =V_{B}\left(\delta_{B}^{2}+\delta_{S}^{2}-2 \delta_{d B} \delta_{S}-2 \delta_{i n B} \delta_{S}\right)+ \\
& +R T\left(\ln \frac{V_{B}}{V_{S}}+1-\frac{V_{B}}{V_{S}}\right)
\end{aligned}
$$

\section{Определение составляющих параметра растворимости спиртов}

Расчет $\delta, \delta_{d}$ и $\delta_{o r}$. Для растворителей и растворенных соединений суммарные параметры растворимости $\delta$ рассчитывали по уравнению (2) из энтальпий испарения $\left[{ }^{15,16}\right]$ или брали из $\left[{ }^{17}\right]$. Энтальпии испарения при 293,15 K (табл. 1) рассчитывали, используя корреляцию Ватсона.

Таблица 1

Физико-химические величины спиртов, использованные в расчетах [15-18]

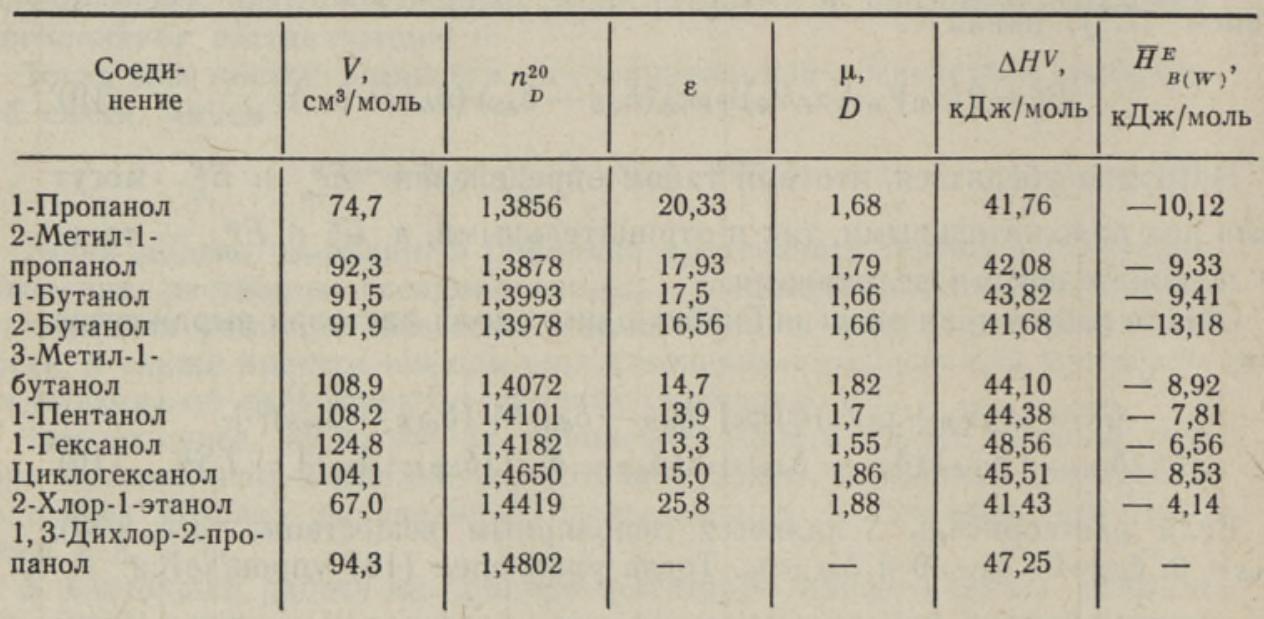

Дисперсионную составляющую параметра растворимости $\delta_{d}$ для полярных соединений рассчитывали по уравнению Келлера из [8]

$$
\delta_{d}=-4,57+108 x-118 x^{2}+45 x^{3},
$$

в котором $x$ обозначает функцию Лоренца-Лоренца $(n-1) /\left(n^{2}+2\right)$, где $n$ - показатель преломления $n_{D}^{20}$ (брали из $\left[{ }^{15,16}\right]$ ).

Ориентационную составляющую параметра растворимости $\delta_{o r}$ pacсчитывали согласно Хансену $\left.{ }^{[1}{ }^{11}\right]$ через показатель преломления, диэлектрическую проницаемость $\varepsilon$ и дипольный момент $\mu$ (брали из $\left.\left[{ }^{15,16}\right]\right)$ :

$$
\delta_{o r}^{2}=\frac{1,2099 \cdot 10^{4}}{V^{2}} \cdot \frac{(\varepsilon-1)\left(n^{2}+2\right)}{\left(2 \varepsilon+n^{2}\right)} \mu^{2} .
$$

Мольные объемы растворителей и растворенных соединений, необходимые для расчетов, получали из справочных данных $\left[{ }^{15,16]}\right.$.

Определение $\delta_{i n}$. Уравнение (14) дает возможность определить вклад индукционного взаимодействия в общую когезионную энергию полярного соединения, если имеются экспериментальные данные о $\gamma_{B(S)}^{\infty}$, а также надежные методы расчета величин $\delta_{d B}$. 


\section{Обозначим}

$$
\left(\delta_{B}^{2}+\delta_{S}^{2}\right) / 2-\delta_{d B} \delta_{S}-T\left(R \ln \gamma_{B(S)}^{\infty}+\bar{S}^{E}\right) / 2 V_{B}=Y,
$$

где $\bar{S} E-$ парциальная избыточная энергия растворения $B$ в $S$.

Тогда уравнение (14) может быть записано в виде

$$
Y=\delta_{\text {in } B} \delta_{S},
$$

т. е. $\delta_{\text {inB }}$ определяется как тангенс угла наклона прямой, изображающей зависимость $Y=f(\delta s)$ для растворов одного $B$ в ряде неполярных $S$.

Для определения величин $Y$ использовали собственные экспериментальные данные о предельных коэффициентах активности спиртов в гексадекане $H\left(\gamma_{B(H)}^{\infty}\right)\left[{ }^{18}\right]$, принятом в качестве «стандартного» неполярного растворителя, и коэффициенты распределения спиртов между несмешивающимися с водой органическими растворителями $S$ и водой $W$ $\left(K_{B(\mathrm{~S} / W)}^{\infty}\right)$, опубликованные в [ $\left.{ }^{20}\right]$. Учитывая, что

$$
K_{B(\mathrm{~S} / W)}^{\infty} \equiv \lim _{x_{B(s) \rightarrow 0}}\left(x_{B(\mathrm{~S})} / x_{B(W)}\right)=\gamma_{B(W)}^{\infty} / \gamma_{B(\mathrm{~S})}^{\infty},
$$

из этих величин рассчитывали предельные коэффициенты активности спиртов в растворителях $S$ :

$$
\ln \gamma_{B(S)}^{\infty}=\ln \left(K_{B(H / W)}^{\infty} / K_{B(S / W)}^{\infty}\right)+\ln \gamma_{B(H)}^{\infty} .
$$

Набор растворителей включал всего 13 соединений: гептан, гексан, октан, нонан, декан, гексадекан, циклогексан, бензол, толуол, ксилолы, этилбензол и $\mathrm{CCl}_{4}$.

Сравнение избыточных энтропий растворения соединений в гексадекане, рассчитанных по Флори-Хаггинсу и полученных из эксперимента $\left[{ }^{19}\right]$, показало, что эти величины существенно расходятся. Авторам это явление представляется вполне естественным, поскольку расчет энтропии по Флори-Хаггинсу дает оценку лишь той части энтропии, которая обусловлена различием в размерах растворенного вещества и растворителя, но не отражает энтропию, вызванную сольватацией и разложением ассоциатов молекул спирта. Поэтому при расчете величин $Y$ для растворенных спиртов вместо расчетных энтропий Флори-Хаггинса (уравнение (12)) использовали экспериментальные значения энтропии того же соединения в гексадекане $\left(\bar{S}_{H}^{E}\right)$, исправленные на разность величин избыточной энтропии по Флори-Хаггинсу в данном растворителе $S\left(\bar{S}_{F S}^{E}\right)$ и гексадекане $H\left(\bar{S}_{F H}^{E}\right)$ :

$$
\bar{S}^{E}=\bar{S}_{H}^{E}+\left(\bar{S}_{F S}^{E}-\bar{S}_{F H}^{E}\right) .
$$

Сопоставление рассчитанных таким образом величин $Y$ для одного спирта в ряде неполярных $S$ с параметрами $\delta s$ показало, что эти величины действительно пропорциональны (коэффициент корреляции $r$ порядка 0,94$)$. Это значит, что уравнение (17) может быть использовано для определения $\delta_{\text {in }}$. ментом

С другой стороны, согласно $\left[{ }^{8,12}\right] \delta_{\text {or }}$ и $\delta_{\text {in }}$ связаны с дипольным мо-

$$
\begin{gathered}
\delta_{i n}=C_{i n} \mu^{2} / V, \\
\delta_{o r}=C_{o r} \mu / V
\end{gathered}
$$

или

$$
\delta_{\text {in }}=\left(C_{\text {in }} / C_{\text {or }}^{2}\right) V \delta_{o r}^{2},
$$

где $C_{\text {in }}$ и $C_{\text {or }}$ - эмпирические константы. 
Сопоставление полученных изложенным выше способом значений $\delta_{\text {in }}$ c $V \delta_{\text {or }}$ для спиртов показывает, что между этими величинами действительно соблюдается линейная зависимость. При этом коэффициент пропорциональности $C \equiv C_{i n} / C_{\text {or }}^{2}$ равен $(1,17 \pm 0,25) \cdot 10^{-3}$, что в пределах точности эксперимента согласуется с величиной $C=(1,11 \pm 0,13)$ $\cdot 10^{-3}$, полученной в $\left[{ }^{14}\right]$ для сложных эфиров. Следует также указать, что некоторое различие в величинах $C$ обусловлено различным способом учета вклада энтропии: в $\left.{ }^{14}\right]$ избыточную энтропию, входящую в уравнение (11), рассчитывали чисто теоретическим путем, а в настоящей работе ввели «исправленные» по уравнению (19) экспериментальные значения $\bar{S}_{H}^{E}$.

Определение $\delta_{a}$ и $\delta_{b}$. Если известны $\delta, \delta_{d}, \delta_{o r}$ и $\delta_{i n}$, по уравнению (2) легко рассчитать вклад водородной связи $\left(2 \delta_{a} \delta_{b}\right)$ в общую когезионную энергию чистой жидкости:

$$
2 \delta_{a B} \delta_{b B}=\delta_{B}^{2}-\left(\delta_{d B}^{2}+\delta_{o r B}^{2}+2 \delta_{i n B} \delta_{d B}\right) .
$$

Тогда проблема определения $\delta_{a}$ и $\delta_{b}$ для отдельных соединений в принципе сводится к установлению шкалы «кислотности» (протонодонорной способности) и «основности» (протоноакцепторной способности) по отношению к произвольно выбранному стандарту. В $\left[{ }^{8,12}\right]$ стандартом выбраны алифатические спирты, для которых принято $\delta_{a}=\delta_{b}$. Нами выбрана в качестве стандарта вода, для молекулы которой $\delta_{b}^{2} / \delta_{a}^{2}$ определено как соотношение энтальпий процессов присоединения и отщепления протона, равное 0,43 [1'], и известен также вклад водородной связи $\left(2 \delta_{a} \delta_{b}=1523\right.$ Дж/см $\left.{ }^{3}\right)$ в $\delta$.

С другой стороны, согласно [ $\left.{ }^{11}\right]$ парциальная энтальпия смешения соединения с водой $\left(\bar{H}_{B(W)}^{E}\right)$ (табл. 1$)$, измеренная экспериментальным путем, должна быть рассмотрена как результат несимметричного взаимодействия, обусловленного водородной связью. Тогда

$$
\bar{H}_{B(W)}^{E}=2 V_{B}\left(\delta_{a B}-\delta_{a W}\right)\left(\delta_{b B}-\delta_{b W}\right) .
$$

Однако эта концепция, по замыслу ее авторов, пренебрегает энергией индукционного взаимодействия между молекулами воды и растворенного соединения. Поэтому уравнение (24) более обосновано записать в виде

$$
\bar{H}_{B(W)}^{E}=2 V_{B}\left[\left(\delta_{i n B}-\delta_{i n W}\right)\left(\delta_{d B}-\delta_{d W}\right)+\left(\delta_{a B}-\delta_{a W}\right)\left(\delta_{b B}-\delta_{b W}\right)\right] .
$$

Тогда $\delta_{a B}$ и $\delta_{b B}$ можно получить путем решения системы уравнений (23) и (25).

Результаты расчета, полученные при использовании парциальных энтальпий смешения из $\left[{ }^{18}\right]$ и величин $\delta_{d w}=12,89, \quad \delta_{\text {or }}=31,41, \quad \delta_{\text {in }}=$ $=20.83^{* *}, \delta_{a w}=34,07$ и $\delta_{b w}=22,34$ для воды $\left(\right.$ все в $\left.\left(Д ж / \mathrm{cm}^{3}\right)^{0,5}\right)$, приведены в табл. 2.

Необходимо также указать, что расчеты $\delta_{a}$ и $\delta_{b}$, проведенные с использованием для $\bar{H}_{B(W)}^{E}$ зависимости (24), дают для спиртов величины $\delta_{a}$, в среднем на $7 \%$ меньшие, и $\delta_{b}-$ на $7 \%$ большие, чем в табл. 2 .

Знание величин $\delta_{a}$ и $\delta_{b}$ для растворенного соединения в принципе дает возможность по предельным коэффициентам активности определять $\delta_{a}$ и $\delta_{b}$ для полярных растворителей. Тем не менее при этом не следует ожидать большой точности, если в таких системах нет досто-

** Получено по уравнению (22) с учетом $C_{i n} / C_{o r}^{2}=1,17 \cdot 10^{-3}$. 
Составляющие параметра растворимости спиртов, (Дж/см $\left.{ }^{3}\right)^{0,5}$

\begin{tabular}{l|c|c|c|c|c|c}
\hline \multicolumn{1}{c|}{ Соединение } & $\delta$ & $\delta_{d}$ & $\delta_{\text {or }}$ & $\delta_{\text {in }}$ & $\delta_{a}$ & $\delta_{b}$ \\
\hline 1-Пропанол & 25,04 & 14,90 & 6,75 & 4,51 & 4,78 & 23,53 \\
2-Метил-1-пропа- & 23,10 & 14,98 & 5,79 & 3,41 & 3,81 & 22,81 \\
нол & 23,83 & 15,35 & 5,43 & 3,09 & 4,60 & 22,60 \\
1-Бутанол & 22,78 & 15,21 & 5,38 & 3,21 & 3,45 & 23,35 \\
2-Бутанол & 22,78 & 15,54 & 4,97 & 2,34 & 4,08 & 22,08 \\
3-Метил-1-бутанол & 22,84 & 15,69 & 4,65 & 2,89 & 3,73 & 21,87 \\
1-Пентанол & 22,10 & 15,86 & 3,67 & 1,67 & 3,97 & 21,54 \\
1-Гексанол & 22,72 & 17,27 & 5,40 & 2,94 & 2,06 & 21,17 \\
Циклогексанол & 26,58 & 16,63 & 8,20 & 6,45 & 3,43 & 21,59 \\
2-Хлор-1-этанол & \multirow{2}{*}{ 3-Дихлор-2-про- } & 22,76 & 17,68 & $6,67^{* *}$ & 4,90 & \multicolumn{2}{|c}{$99,7^{*}$}
\end{tabular}

* Определена как $2 \delta_{a} \delta_{b}$, Дж/см³.

** Рассчитана по уравнению (22) через $\delta_{i n}$.

верной экспериментальной оценки парциальной избыточной энтропии растворенного соединения. По мнению авторов, расчет энтропии по Флори-Хаггинсу, используемый многими авторами, в таких системах вряд ли обоснован.

\section{Выводы}

1. Предложен метод разделения суммарного параметра растворимости самоассоциированного соединения на дисперсионную, ориентационную, индукционную, протонодонорную и протоноакцепторную составляющие исходя из предельных коэффициентов активности в неполярных растворителях и из парциальных энтальпий растворения в воде.

2. На основе собственных экспериментальных данных определены составляющие параметра растворимости для 10 спиртов.

\section{ЛИ Т Е РА Т У РА}

1. Hildebrand, J. H., Prausnitz, J. M., Scott, R. L. Regular and Related Solutions. New York, 1970.

2. Funk, E. W., Prausnitz, J. M. Thermodynamic properties of liquid mixtures: aromatic saturated hydrocarbon systems. - Ind. Eng. Chem., 1970, 62, N 9, 8-15.

3. Weimer, R. F., Prausnitz, J. M. Screen extraction solvent this way. - Hydrocarbon Process, 1965, 14, N 9, 237-242.

4. Blanks, R. F., Prausnitz, J. M. Thermodynamics of polymer solubility in polar and nonpolar systems. - Ind. Eng. Chem. Fundam., 1964, 3, N 1, 1-8.

5. Аарна А., Арро Я., Мэлдер Л., Тамвелиус Х. Разделение параметра растворимости на составляющие и определение коэффициентов активности самоассоцинрующихся веществ по данным распределения между двумя жидкими фазамн. Изв. АН ЭССР. Хим. Геол., 1975, 24, № 2, 115-122.

6. Арро Я. В., Мэлдер Л. И. Определение коэффициентов активности двухатомных фенолов в воде по данным распределения между двумя жидкими фазами. Ж. физ. хим., 1975, 49, № 4, $1077-1079$.

7. Barton, A. F. M. Solubility parameters. - Chem. Revs., 1975, 75, N 6, 731-753.

8. Karger, B. L., Snyder, L. R., Eon, $C$. An expanded solubility parameter treatment for classification and use of chromatographic solvents and adsorbents. - J. Chromatogr., 1976, 125, 71-88.

9. Milanova, E., Cova, G. C. B. Limiting activity coefficients in dilute solutions of nonelectrolytes. I. Determination for polar-nonpolar binary mixtures and a solubilityparameter treatment of results. - Can. J. Chem., 1982, 60, N 21, 2697-2706. 
10. Keller, R. A., Karger, B. L., Snyder, L. R. Use of the solubility parameter in predicting chromatographic retention and eluotrophic strength. - Gas Chromatogr., Proc. Int. Symp. (Europe), 1971, 8, N 125, 125-140.

11. Tijssen, R., Billiet, H. A. H., Schoenmakers, P. J. Use of the solubility parameter for predicting selectivity and retention in chromatography. - J. Chromatogr., 1976, 122, 185-203.

12. Karger, B. L., Snyder, L. R., Eon, C. Expanded solubility parameter treatment for classification and use of chromatographic solvents and adsorbents. - Anal. Chem., 1978, 50, N 14, 2126-2136.

13. Мэлдер Л., Эббер А. Разделение суммарного параметра растворимости полярных растворителей без Н-связи на днсперсионную, ориентационную и индукционную составляющие. - Изв. АН ЭССР. Хим., 1981, 30, № 1, 39-43.

14. Мэлдер Л. Н., Эббер А. В. Определение ориентационного и индукционного компонентов параметра растворимости полярных соединений без Н-связи. - Ж. прикл, хим., 1982, 55, № 9, 1979-1983.

15. Handbook of Chemistry and Physics. 57th ed. Cleveland-Ohio, 1976-1977.

16. Lange's Handbook of Chemistry. 12th ed. New York-St. Louis, et al., 1979.

17. Hoy, $K . L$. New values of the solubility parameters from vapor pressure data. J. Paint Technol., 1970, 42, N 541, 76-117.

18. Белоусов В. П., Панов М. Ю. Термодинамика водных растворов неэлектролитов. Л., 1983.

19. Игнат А., Мельдер Л. Предельные коэффициенты активности и избыточные термодинамические функции некоторых полярных соединений в гексадекане. - Изв. АН ЭССР. Хим., 1985, 34, № 3, 201-205.

20. Нгнат А., Мельдер Л. Некоторые закономерности распределения спиртов между фазами органического растворителя и воды, - Изв. АН ЭССР. Хим., 1985, 34, № $1,63-68$.

Таллинский политехнический институт

Поступила в редакцию

Институт химии

22/XI 1985

Академии наук Эстонской ССР

\section{A. IGNAT, L. MOLDER}

\section{AUTOASSOTSIEERUVATE OHENDITE LAHUSTUVUSPARAMEETRI JAOTAMINE KOMPONENTIDEKS}

Artiklis on esitatud meetod autoassotsieeruvate ühendite summaarse lahustuvusparameetri jaotamiseks dispersiooni- $\left(\delta_{d}\right)$, orientatsiooni- $\left(\delta_{o r}\right)$, induktsiooni- $\left(\delta_{i n}\right)$, prootondonoorseks $\left(\delta_{a}\right)$ ja prootonaktseptoorseks $\left(\delta_{b}\right)$ komponendiks. Kasutatakse ühendite eksperimentaalselt määratud jaotuskoefitsiente mittepolaarsete lahustite ja vee vahel, partsiaalseid lahustuvusentalpiaid vees ning aktiivsustegureid heksadekaanis kui standardses mittepolaarses lahustis. On demonstreeritud meetodi kasutatavust alkoholide puhul ning leitud, et nende jaoks $\delta_{i n} / \delta_{o r}{ }^{2} V(V-$ molaarruumala $)$ on $(1,17 \pm 0,25) \cdot 10^{-3}$.

\section{A. IGNAT, L. MOULDER}

\section{SPLITTING THE SOLUBILITY PARAMETER OF SELF-ASSOCIATING SUBSTANCES INTO COMPONENTS}

A procedure of calculating dispersion $\left(\delta_{d}\right)$, orientation $\left(\delta_{o r}\right)$, induction $\left(\delta_{i n}\right)$, proton donor $\left(\delta_{a}\right)$ and proton acceptor $\left(\delta_{b}\right)$ solubility parameter components has been proposed. The method is based on experimental data of the partition of self-associating substance at infinite dilution between a series of non-polar solvents and water, on data of enthalpy of solution in water and on data of activity coefficients of the partitioned substances in hexadecane (standard non-polar solvent). The use of the presented treatment has been demonstrated on an example of alcohols. It has been found that $\delta_{i n} / \delta_{o r}{ }^{2} V$ (where $V$ is the molar volume) for alcohols is equal to $(1.17 \pm 0.25) \cdot 10^{-3}$. The solubility parameter components for 10 alcohols have been tabulated. 